Gynäkologe 2019·52:718-719

https://doi.org/10.1007/s00129-019-04500-6

(c) Springer Medizin Verlag GmbH, ein Teil von Springer Nature 2019

\author{
Klaus Vetter $\cdot$ Roland Zimmermann ${ }^{2}$ \\ 'Berlin, Deutschland \\ ${ }^{2}$ Klinik für Geburtshilfe, UniversitätsSpital Zürich, Zürich, Schweiz
}

\title{
Supportive Therapien in der Schwangerschaft
}

\section{Schwangerschaft zwischen Natur pur und pharmazeutischem Ereignis}

Dem unübersehbaren Trend, tierisches Eiweiß nicht mehr zur Nahrungsgrundlage machen $\mathrm{zu}$ wollen, ist der Artikel von Edith Gätjen zur veganen Ernährung in der Schwangerschaft geschuldet. Sie zeigt sehr praktisch auf, wie trotz bewusster subjektiver Beschränkungen des Nahrungsumfangs eine Schwangerschaft ohne jegliche Mangelerscheinungen unproblematisch gestaltbar ist. Rezeptvorschläge und eine vegane Ernährungspyramide stellen eine sehr nützliche Unterstützung des Texts dar.

\section{\) Auch bei veganer \\ Ernährung lässt sich eine Schwangerschaft ohne jegliche Mangelerscheinung gestalten}

Ein zunehmendes Problem stellen Schwangerschaften nach unterschiedlichsten bariatrischen Operationen dar. Diese haben nicht nur den Effekt verminderter Kalorienaufnahme mit konsekutivem chronischem Hungerzustand, sondern führen auch, je nach Methode, zu eingeschränkter Absorption essenzieller Nährstoffe infolge postoperativer Veränderungen der intestinalen Absorptionsfläche. Damit kann es zu Mangelzuständen bei Mikronährstoffen kommen, die für die Mutter relativ einfach überbrückbar sind, aber für das Ungeborene erhebliche unerwünschte Folgen haben können. Entsprechend muss prä- und postoperativ individuell beraten sowie in einer Schwangerschaft ganz gezielt überwacht und therapiert werden. Bernd Schultes hat sich dem Thema der supportiven Therapie nach Adipositaschirurgie nicht nur wissenschaftlich, sondern auch sehr praktisch gewidmet und zeigt Wege zur wichtigen Problemvermeidung auf.

Durch einen CME(Continuing $\mathrm{Me}$ dical Education)-Artikel zu Schilddrüsenhormonsubstitution in der Schwangerschaft von Timo Minnemann und $\mathrm{Ca}$ tharina Bullmann wird dieses Themenheft komplettiert. Sie machen deutlich, wie essenziell die Schilddrüse und ihre Störungen auf das Schwangerschaftsergebnis einwirken können. Grundlagen sind, erstens, dass Thyroxin entgegen früheren Annahmen reguliert in geringen Mengen von der Mutter zum Feten transferiert wird, zweitens, dass Jodid $\left(\mathrm{I}^{-}\right)$durch Natrium-Jodid-Symporter (NIS) diaplazentar transportiert wird, und, drittens, dass Autoantikörper gegen Schilddrüsengewebe plazentagängig sind. Sie können den Schwangerschaftsverlauf unterschiedlich stark beeinflussen, bis hin zu Abort, Plazentainsuffizienz oder Frühgeburt; Auswirkungen auf den Fetus sind möglich. Die therapeutischen Konsequenzen sind entsprechend: Ein Jodmangel soll ausgeglichen werden. Eine Hypothyreose ist durch Thyroxin zu behandeln - auch im Sinn der neurologischen Entwicklung des Feten. Ein spezifisches Konzept bei Vorliegen von Schilddrüsenautoantikörpern oder zur Behandlung einer Hashimoto-Thyreoiditis in der Schwangerschaft gibt es nicht. Bisher weisen Studien bei den vorgeschlagenen Therapien keine negativen Effekte für den Nachwuchs auf. 
Wir hoffen, mit dem vorliegenden Heft zur entspannten Beratung bei einigen aktuell teils kontrovers diskutierten Fragestellungen in der Schwangerschaft beizutragen.

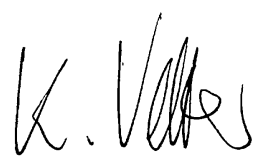

Klaus Vetter

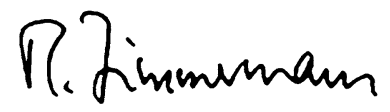

\section{Roland Zimmermann}

\section{Korrespondenzadresse}

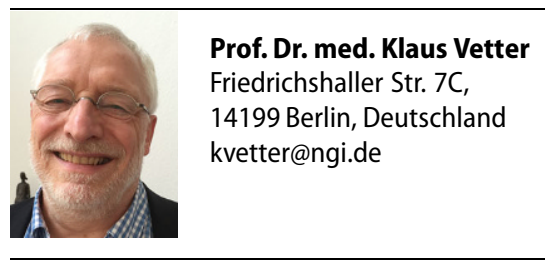

Interessenkonflikt. K. Vetter und R. Zimmermann geben an, dass kein Interessenkonflikt besteht.

\section{G. Gille}

\section{Mädchen fragen, Mütter wissen \\ Das Infobuch für Mütter von Mädchen ab 11 Jahren}

Berlin Heidelberg: Springer-Verlag 2019, 1, 164 S., 73 farbige Abb., (ISBN: 978-3662-58448-4), Softcover 19,99 EUR

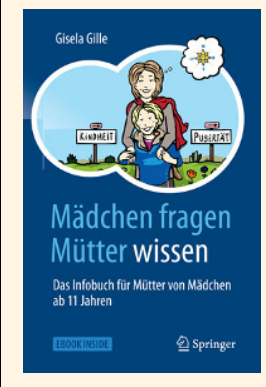

Mädchen sollten möglichst frühzeitig, also zu Beginn der Pubertät, die Gelegenheit haben, sich objektiv und gleichzeitig liebevoll übermittelte Informationen über ihren sich entwi-

ckelnden Körper einzuholen. Sensibilität und Verständnis für Veränderungen und Signale des Mädchenkörpers sollen geweckt werden und damit praktische Kompetenz für die daraus resultierende Verantwortung. Denn nur Mädchen, die mit den faszinierenden Abläufen in ihrem Körper vertraut sind und wissen, was auf sie zukommt, können "ja“ sagen zu sich selber und können dann auch "nein" sagen zu Dingen, die sie nicht wirklich wollen.

Allen Umfragen zufolge gelten Mütter für ihre Töchter in der Pubertät als präferierte Personen zur Sexualaufklärung. Mütter sind für Mädchen in der frühen Pubertät auch deshalb besonders glaubhaft, weil der Wissenstransfer eine Gemeinsamkeit herstellt: Beide sind betroffen und beide wissen, wovon sie sprechen, Verständnis- und Vertrauensbrücken entstehen von selbst. Mütter sollten es sich nicht nehmen lassen, erstrebenswertes Verhalten und wünschenswerte Einstellungen entscheidend mitzuprägen. Solche Gespräche können zu den Sternstunden einer Mutter-Tochter-Beziehung gehören.

Aber Mütter können auch nur das weitergeben, was sie wissen und was sie als Frau repräsentieren. Deshalb ist die Pubertät der heranwachsenden Tochter für die Mutter auch ein Anlass zur Reflexion eigener Vorstellungen von Weiblichkeit, des eigenen Verhältnisses zum Körper und zur Sexualität und vielleicht auch zu eigenen Tabuisierungen.

Die Ärztin Dr. Gisela Gille hat jetzt aus ihrer jahrzehntelangen Erfahrung in der Aufklärungsarbeit mit jungen Mädchen auch ein Buch für deren Mütter geschrieben, das ei- ne wertschätzende Auseinandersetzung mit dem weiblichen Körper und der weiblichen Sexualität ermöglicht und das ein Plädoyer für "Ich bin gut so wie ich bin" sein möchte: Es geht dabei um die spannenden, aktuellen Erkenntnisse zur Pubertät, um die Körperakzeptanzprobleme von Mädchen, um die faszinierenden Abläufe im weiblichen Körper im Kontext der Fruchtbarkeit, um den weiblichen Zyklus, um das erste Verliebtsein und altersentsprechend geht es auch um die erwachende weibliche Sexualität - ein Thema, das früher ein "No go" war und das heute als "Everything goes" viele Mädchen überfordert. Auch die Angebote in unseren Praxen und in speziellen Mädchensprechstunden werden erklärt und empfohlen.

Ein Buch also, auf das Mütter gewartet haben, das selbstverständlich auch Väter von pubertierenden Töchtern mit Gewinn lesen können und das wir unseren Patientinnen mit Töchtern in der Pubertät wärmstens empfehlen sollten.

K. Schaudig (Hamburg) 